Article

\title{
Green Synthesis of Potent Antimicrobial Silver Nanoparticles Using Different Plant Extracts and Their Mixtures
}

\author{
May Reda ${ }^{1}$, Akram Ashames ${ }^{1, * \mathbb{D}}$, Zehra Edis ${ }^{1}$, Samir Bloukh ${ }^{2}$, Richie Bhandare ${ }^{1}$ and \\ Hamed Abu Sara ${ }^{2}$ \\ 1 Department of Pharmaceutical Sciences, College of Pharmacy and Health Sciences, Ajman University, \\ P.O. Box 346, Ajman, UAE \\ 2 Department of Clinical Sciences, College of Pharmacy and Health Sciences, Ajman University, P.O. Box 346, \\ Ajman, UAE \\ * Correspondence: a.ashames@ajman.ac.ae
}

Received: 21 June 2019; Accepted: 2 August 2019; Published: 4 August 2019

\begin{abstract}
Nano-sized metals have been introduced as a promising solution for microbial resistance to antimicrobial agents. Silver nanoparticles (AgNPs) have been proven to possess good antimicrobial activity. Green synthesis of AgNPs has been reported as safe, low cost and ecofriendly. This methodology uses extracts originating from different plants to reduce silver ions from $\mathrm{AgNO}_{3}$ into nano-sized particles. In this study, extracts of several plants including ginger, garlic, capsicum and their mixtures were successfully used to produce AgNPs. Numerous spectroscopic, light scattering and microscopic techniques were employed to characterize the synthesized AgNPs. Agar well diffusion assay was performed to investigate the antimicrobial activity of AgNPs. The biosynthesized AgNPs have spherical shape with a size range of 20-70 nm. Garlic extract, pure or in mixture with ginger extract, generated AgNPs of the smallest size. The presence of the plant-origin capping agents surrounding AgNPs was proven by Fourier-transform infrared spectroscopy. The AgNPs, at a concentration of $50 \mu \mathrm{g} / \mathrm{mL}$, demonstrated potent antimicrobial activity against Staphyloccocus aureus, Escherichia coli and Candida albicans as indicated by the zones of inhibitions. Our results revealed that AgNPs having potent antimicrobial activity could be prepared using different pure plant extracts and their mixtures.
\end{abstract}

Keywords: AgNPs; green synthesis; plant extract; chemical methods; antibacterial; antifungal

\section{Introduction}

Microbial resistance to antimicrobial agents has become a serious concern worldwide, leading to an increase in mortality in most cases [1]. Therefore, finding new alternatives to the currently used antibiotics has become a necessity. Nano-sized metals have been shown to have a good antimicrobial effect and they can be introduced as a solution for the resistance problem. Moreover, nanometals have diverse applications, which can be explained by attaining a high surface area to volume ratio [1]. Researchers mainly focus on AgNPs as they have potent antimicrobial activity [2,3]. In ancient times, silver was used as an antiseptic and antimicrobial agent to treat different types of infectious diseases due to its low cytotoxicity [4]. AgNPs were used to inhibit the growth of bacterial biofilms [5]. Further studies proved that AgNPs also possess antifungal activity [6]. However, AgNPs may undergo aggregation and form larger particles, which lowers their potential properties. Therefore, the use of stabilizing and capping agents to surround the surface of AgNPs is very crucial to maintain their stability and keep them active over time [7]. Many research groups have conducted experiments to elucidate the effect of the stabilizing agents on the synthesized AgNPs [8]. In addition to the stabilizing 
agent, other physicochemical parameters that influence the biological activity of AgNPs are size, shape and surface charge [9].

The three methodologies through which AgNPs can be synthesized are chemical, physical and green methods. Lately, a number of research groups have adopted the green plant-based reduction methods which are also considered safe, simple and cost effective protocols [10]. Multiple studies have demonstrated green synthesis of AgNPs using plant extracts such as Cinnamon zeylanicum, Phoenix dactylifera and Terminalia chebula [11-13].

The main advantage of the green method was the presence of naturally occurring biomolecules, such as proteins, enzymes, tannins, phenols, sugars and flavonoids that can be used safely as reducing and stabilizing agents to form stable nanometals [14,15].

In the present work, ginger, garlic and capsicum, which are known to have antimicrobial constituents, were selected to synthesize AgNPs. The naturally occurring biomolecules and metabolites in the abovementioned plants can act as reducing and capping agents that prevent agglomeration by stabilizing the AgNPs.

Capsicum (Capsicum frutescens) - which is known as sweet pepper-contains capsaicin alkaloids and other derivatives [16]. Capsaicin and its derivatives have been extensively investigated in the field of antimicrobial drugs [17]. Ginger (Zingiber officinale) contains various bioactive components, such as alkaloids, flavonoids and zingiberene, which possess antioxidant properties [18]. In addition, ginger has been used as a medicinal plant in traditional cultures for the treatment of a number of diseases. Ginger extracts have shown good antibacterial activity against a number of pathogens such as Staphylococcus aureus, Staphylococcus pneumonia, as well as Hemophilus influenza [19]. Garlic (Allium sativum) has also been shown to have antimicrobial and antioxidant activities $[19,20]$. Researchers have demonstrated that active compounds in garlic, such as allyl disulfide and allyl cysteine, are protective against free radical damage. In addition, garlic extract was reported as a good bio-reducing agent for AgNPs synthesis [21].

The present work demonstrates the use of pure plant extracts and mixtures of two extracts to prepare AgNPs. The plants used in this study were selected considering their reducing ability needed for the reduction of silver ions from $\mathrm{AgNO}_{3}$ solution. In addition, these plants were known to have antimicrobial compounds as well. AgNPs were characterized and their antimicrobial activity was evaluated extensively.

\section{Materials and Methods}

\subsection{Chemical, Plants, Microorganism Strains and Media}

Finely ground, dried powders of capsicum, ginger and garlic plants were purchased from the local market in Ajman, UAE. Silver nitrate $\left(\mathrm{AgNO}_{3}\right)(>99 \%)$ was purchased from Sigma-Aldrich (Munich, Germany) and was used as received. Deionized (DI) water was used for all the processes. Disposable sterilized Petri dishes with Mueller Hinton agar and bacterial strain S. aureus ATCC 25923 were procured from Liofilchem Roseto degli Abruzzi (Italy). E. coli WDCM (World Data Centre for Microorganisms) 00013 Vitroids and C. albicans WDCM 00054 Vitroids was purchased from Sigma-Aldrich in St. Louis, MO, USA.

\subsection{Preparation of Plant Powder Aqueous Extract}

Ginger, garlic and capsicum extracts were prepared by adding $19 \mathrm{~g}$ of the dried plant powder to $100 \mathrm{~mL}$ deionized (DI) water. The mixture was stirred for $15 \mathrm{~min}$ at $55{ }^{\circ} \mathrm{C}$. The extracts were then cooled and filtered through Whatman grade 1 filter paper. The filtrate was stored in a sealed storage tube and stock solutions of the extracts were kept in refrigerator. 


\subsection{Biosynthesis of AgNPs by Using the Plant Extracts}

Aqueous extract of plants were used as reducing agent in the green synthesis of AgNPs. The effect of extract, $\mathrm{AgNO}_{3}$ concentration, time and temperature of reaction were standardized to obtain the optimum values for the synthesis of AgNPs. The stock solutions of the extracts were diluted to 5,10 and $20 \%(v / v)$. The extracts of ginger (Gin), garlic (Gar) and capsicum (Cap) as well as the mixtures of these extracts, including Gin-Gar, Gin-Cap and Gar-Cap, were used as reducing agents for the $\mathrm{AgNO}_{3}$ solution. All the mixtures of the plant extracts were prepared in the ratio 1:1 by volume. Two milliliters of $(1,2.5,5$ and $10 \mathrm{mmol} / \mathrm{L})$ of the $\mathrm{AgNO}_{3}$ solution was added to the working plant extract solution drop by drop under heating $\left(55^{\circ}\right)$ for $25 \mathrm{~min}$ and with vigorous stirring. The yellowish solution of the diluted plant extract changed to yellowish-brown colloidal dispersion after the addition of the $\mathrm{AgNO}_{3}$ solution, indicating the formation of AgNPs. Optimum results were achieved with concentrations of $5 \%(v / v)$ extracts and $10 \mathrm{mmol} / \mathrm{L} \mathrm{AgNO}$ solution. After the synthesis of AgNPs, the solution containing nanoparticles was centrifuged at $8000 \mathrm{rpm}$ for $10 \mathrm{~min}$ to extract AgNPs from the solution. The sediment of AgNPs was used for analysis. The dried AgNPs were kept in a microtube in the refrigerator for further study.

\subsection{Characterizations of Synthesized AgNPs}

\subsubsection{Morphological Examination and Particle-Size Measurement}

AgNPs samples were studied by scanning electron microscopy-energy-dispersive X-ray spectroscopy (SEM-EDX), model VEGA3 from Tescan (Brno, Czech Republic). A drop of AgNPs dispersion were placed with distilled water onto a carbon-coated copper grid and then left to dry under ambient conditions. EDX analysis was carried out to detect the presence of elemental silver.

\subsubsection{Size and Zeta-Potential Analysis}

The average size and size distribution of the AgNPs were measured and the polydispersity index (PDI) was calculated. Size and size distribution analyses were conducted by DLS, model SZ-100 purchased from Horiba (Palaiseau, France) using scattered light at 90 or 173 degrees angles, automatically selected by the apparatus. The zeta potential measurement was performed at $25^{\circ} \mathrm{C}$ to investigate the dispersion and stability of AgNPs.

\subsubsection{UV-Vis Spectrophotometry}

Ultraviolet-visible (UV-Vis) spectrophotometry is the most important and simple technique to confirm the formation of nanoparticles. The absorbance spectrum of different samples was recorded using a UV-Vis spectrophotometer model 1800 from Shimadzu (Kyoto, Japan) using the wavelength range from 190 to $800 \mathrm{~nm}$.

\subsubsection{Fourier-Transform Infrared Spectroscopy}

AgNPs from all the plant extracts were freeze-dried and then analyzed at $500-4000 \mathrm{~cm}^{-1}$ using a Fourier transform infrared (FTIR) spectrometer (Shimadzu, Koyoto, Japan). FTIR analysis was performed to classify the biomolecules in the plant extracts surrounding the nanoparticles.

\subsubsection{In Vitro Antimicrobial Activity of AgNPs}

The antimicrobial well diffusion assays were performed against the three pathogenic microbial strains, Staphyloccocus aureus, Escherichia coli and Candida albicans. All the selected strains were inoculated in Mueller Hinton Broth (MHB) cultures. A loopful of pathogenic cells from the stock cultures were transferred to test tubes containing $10 \mathrm{~mL}$ MHB and incubated at $37^{\circ} \mathrm{C}$ for $24 \mathrm{~h}$. Agar well diffusion method was carried out according to the standard method to assess the antibacterial activity of the synthesized AgNPs [16]. The standard inoculum using microbial suspensions containing $10^{5}$ 
colony-forming units (CFU) per mL were swabbed on Muller Hinton agar (MHA) and allowed to dry for $10 \mathrm{~min}$. The assay was performed for each solution containing the AgNPs from Gin, Gar, Cap, Gin-Gar, Gin-Cap and Gar-Cap as well as the control. The concentration of the nanoparticles used in the samples was $50 \mu \mathrm{g} / \mathrm{mL}$. Wells of about $6 \mathrm{~mm}$ diameter were made aseptically using a gel puncture instrument and $70 \mu \mathrm{L}$ of each solution was placed in each separate well. Finally, the inoculated plates for all the strains were incubated at $37^{\circ} \mathrm{C}$ for $24 \mathrm{~h}$. Antibacterial activity was evaluated by measuring the diameter of the zones of inhibition (ZOIs) around the well, measured to the nearest millimeter with a ruler. An absence of inhibition zone indicates no antimicrobial activity. The experiments were replicated three times and the results represent the average of three independent experiments.

\subsubsection{Statistical Analysis}

All ZOI values are expressed as means ( \pm standard deviation). The results were analyzed by one-way analysis of variance (ANOVA) Tukey-Kramer multiple-comparison test. The $p$ value of $<0.001$ was considered to be significant. The software SPSS (SPSS Inc, Chicago, IL, USA) was employed for the statistical analysis.

\section{Results and Discussion}

Experimental conditions were optimized by conducting several runs of green synthesized AgNPs using microorganisms and plants extracts as reported in the literature $[10,22]$. The green synthesis of nanoparticles can be accomplished from either plants or microorganisms; however, many research groups use plants for the effective synthesis of AgNPs because of the simple and one-step method that does not require culturing or purification. The use of Calophyllum tomentosum and Rhinacanthus nasutus leaf extracts for the preparation of AgNPs in large yields has also been reported [23,24].

In the present study, AgNPs were synthesized using extracts of Gin, Gar, Cap, Gin-Gar, Gin-Cap and Gar-Cap. The last three samples are mixtures of two plant extracts each. In this study, reduction of silver nitrate ions $\left(\mathrm{Ag}^{+}\right)$to $\mathrm{Ag}^{0}$ was achieved rapidly within $30 \mathrm{~min}$ of the incubation period. Zero Valente of silver metal aggregated around the bioactive compounds, present in the extracts, to form nanoparticles. Moreover, it was confirmed by color change of the reaction solution from watery yellowish to colloidal brownish (Figure 1) [1]. This color change is a morphological indicator for the formation of colloidal solution of AgNPs. The color of silver colloid is endorsed to surface plasmon resonance (SPR) because of the collective oscillation of free conduction electrons caused by an interacting electromagnetic field [22]. The plant extract solutions were used at a dilution of $5 \%$ as further dilution resulted in lower yield of AgNPs.

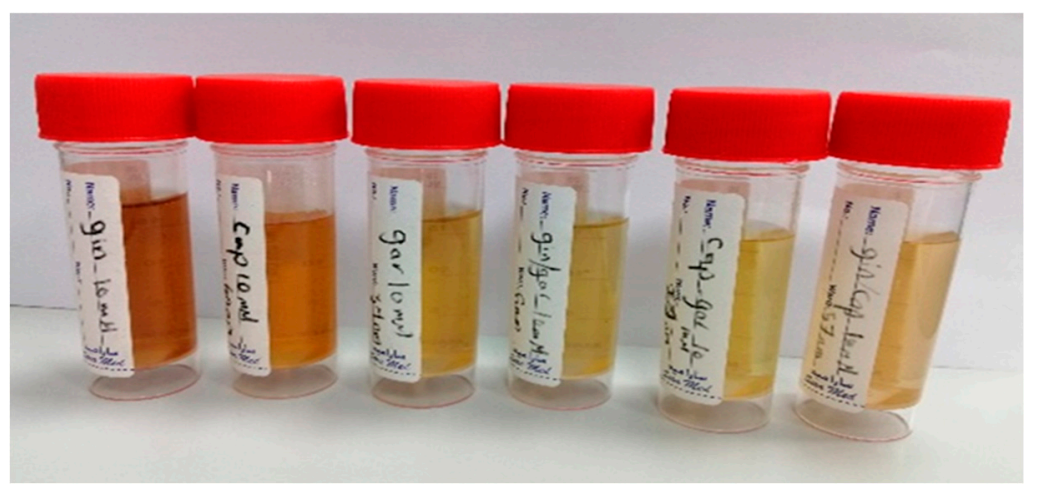

Figure 1. AgNPs in plant solutions (Gin, Gar, Cap, Gin-Gar, Gin-Cap and Gar-Cap) in the same extract concentration.

Interestingly, stable AgNPs were formed by using Gar, Gin, Cap and the three plant extract mixtures as stabilizing agents for AgNPs instead of chemical stabilizers such as polyvinyl alcohol (PVA), polyacrylic acid (PAA), polyethylene glycol (PEG) and mercaptosuccinic acid (MSA) [8]. 


\subsection{UV-Vis Absorbance Spectrophotometry}

Absorption spectroscopy was used to study the optical properties of AgNPs. A characteristic peak at $440 \mathrm{~nm}$ in the UV-Vis spectrum was noticed and it confirms the formation of AgNPs (Figure 2). Surface Plasmon Resonance (SPR) patterns are commonly used as indicative tools for metal nanoparticle formation as SPR depends on a number of parameters, such as size and medium dielectric constant [1]. The SPR band observed at the beginning of particle size formation corresponds to the absorption spectra of spherical nanoparticles. Garlic and ginger extracts are known to contain large amounts of flavonoids and phenolic compounds, which have an essential role in the reduction process during the synthesis of metal nanoparticles [19]. The organosulfur compounds present in the garlic extract may modify the surface chemistry of the AgNPs through thiolate bonding. The spherical shape of AgNPs also depends on single SPR bands, which are evident of the Mie theory [18].

(a)

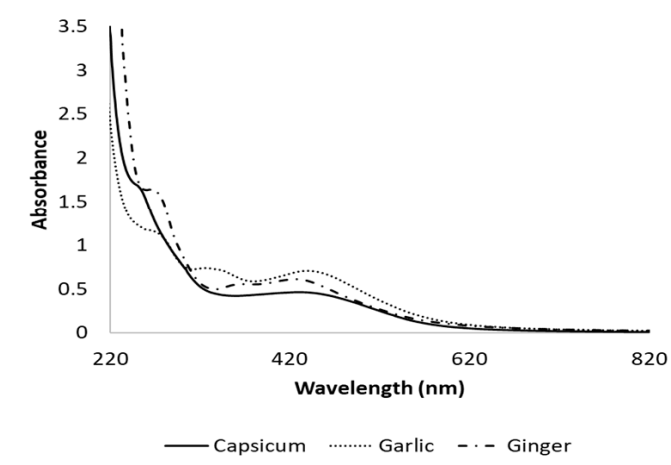

(b)

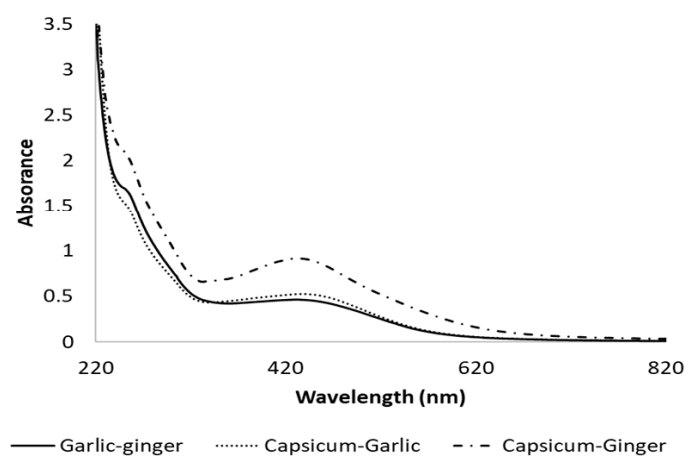

Figure 2. Ultraviolet-visible (UV-Vis) Spectra of AgNPs prepared with (a) Pure plant extract, (b) Mixtures of plant extracts.

Capsaicin alkaloid and antioxidants of the capsicum extract act as reducing and stabilizing agents during the nanoparticle formation. The reduction of silver ions and the growth of nanoparticles happened within one hour due to excitation of surface plasmon vibrations in the nanoparticles [16]. Increasing the extract concentration above $5 \% v / v$ led to formation of a grayish dispersion, indicating the formation of silver colloid due to particle aggregation [1-26]. This can be explained by the high concentration of the bio-reducing agents leading to very small nanoparticles that tend to aggregate and result in the formation of larger stable particles. Broader peaks in the UV-Vis spectra indicate increased polydispersity and/or particle diameter [21]. However, the peaks formed around 250 and $270 \mathrm{~nm}$ corresponded to the compounds naturally found in the plant extracts.

\subsection{Measurement of DLS and Zeta-Potential Analysis}

As shown in Table 1, the pure and mixture extracts gave a narrow particle size distribution and small AgNPs that ranged in sizes from $60 \mathrm{~nm}$ to $19 \mathrm{~nm}$ (Figure 3). The largest sized AgNPs were formed by using the capsicum extract, while the smallest sized AgNPs were formed with the garlic extract.

Table 1. Zeta potential measurements and DLS results for each sample of AgNPs.

\begin{tabular}{cccc}
\hline $\begin{array}{c}\text { Plant Extract Used } \mathbf{( 5 \%} \boldsymbol{v} / \boldsymbol{v}) \\
\text { Silver Nitrate } \mathbf{9 1 0} \mathbf{~ m M}\end{array}$ & $\begin{array}{c}\text { Zeta-Potential } \\
\mathbf{( m V )}\end{array}$ & $\begin{array}{c}\text { Particle Size Mean } \\
\mathbf{( n m )}\end{array}$ & $\begin{array}{c}\text { Poly-Dispersity Index } \\
\text { (PDI) }\end{array}$ \\
\hline Cap & -34.5 & $60.9 \pm 12.2$ & 0.149 \\
Gar & -23.5 & $19.1 \pm 1.2$ & 0.982 \\
Gin & -36.8 & $57.3 \pm 5.5$ & 0.718 \\
Cap-Gar & -18.8 & $56.9 \pm 3.5$ & 0.982 \\
Cap-Gin & -37.1 & $57.4 \pm 3.4$ & 0.788 \\
Gar-Gin & -34.0 & $22.4 \pm 1.0$ & 1.190 \\
\hline
\end{tabular}



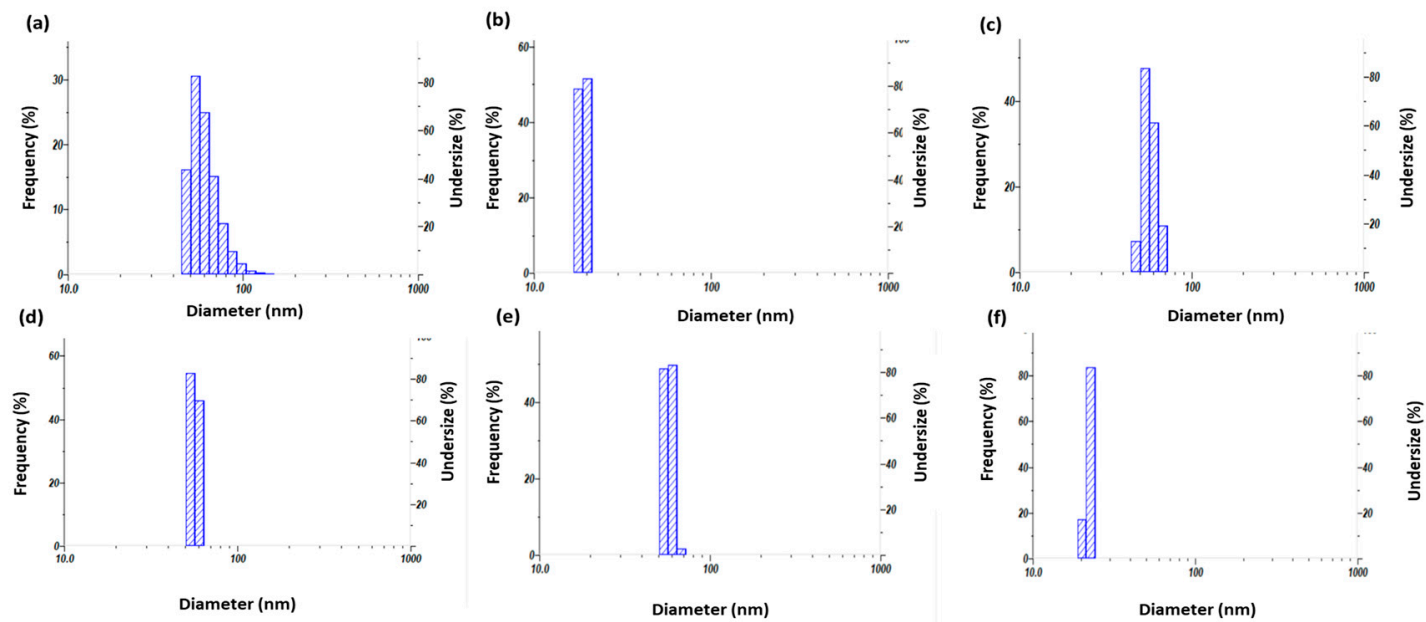

Figure 3. Dynamic light scattering (DLS) analysis of AgNPs: (a) AgNPs with Cap (b) AgNPs with Gar (c) AgNPs with Gin (d) AgNPs with Cap-Gar (e) AgNPs with Cap-Gin (f) AgNPs with Gar-Gin.

The size measured by the nanoparticle nanosize analyzer (DLS) was, to some extent, larger compared with the measurements reported from SEM, which is expected as DLS measures the hydrodynamic size. The zeta potential values for AgNPs were in the expected range of values for all the samples (Figure 4) indicating that the synthesized AgNPs were sensibly stable and well dispersed (Table 1). However, the negative zeta potential value may be due to the capping agents, which mainly consisted of negatively charged groups coating the surface of AgNPs [27]. Regarding the particle-size distribution, all the prepared AgNPs were polydisperse, as revealed by the PDI value. This could be attributed to variations in the growth rates of individual particles during the nucleation step $[28,29]$.

(a)

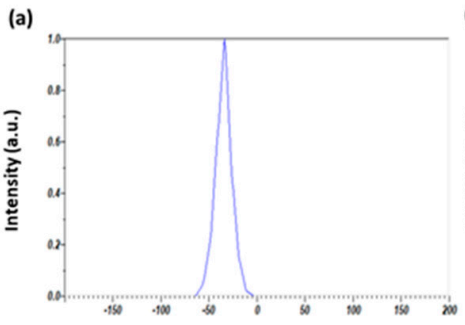

(d)

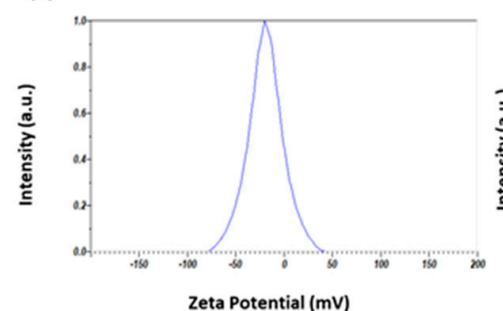

(b)

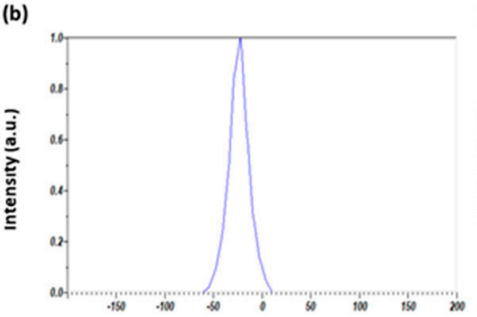

(e)

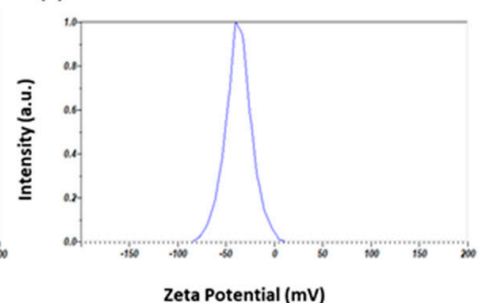

(c)

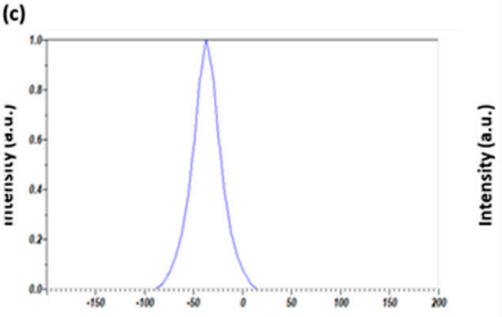

(f)

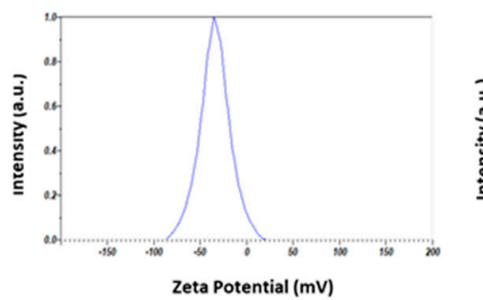

Figure 4. Zeta analysis of AgNPs: (a) AgNPs with Cap (b) AgNPs with Gar (c) AgNPs with Gin (d) AgNPs with Cap-Gar (e) AgNPs with Cap-Gin (f) AgNPs with Gar-Gin.

The Zeta-potential values of AgNPs indicated that stabilization of the synthesized AgNPs by the capping agents was evident, which is in agreement with literature [1]. The negative surface charge is likely due to the adsorption of bioactive components of the plant extract that are distributed on the surface of the AgNPs [1,24]. Generally, the suspensions that exhibit a Zeta potential lower than $20 \mathrm{mV}$ are considered unstable and this results in the precipitation of particles from the solution. Interestingly, it was observed that Zeta-potential values were slightly elevated as the concentration of the extract increases, which is likely because of the presence of negatively-charged capping agents [1,28]. 
Both the concentration and size of the synthesized AgNPs were notably increased by increasing the concentration of the extract in the reaction mixture.

\subsection{Morphological and Size Measurement by SEM}

A number of parameters, such as composition, shape, size and size distribution, could have a pronounced effect on the antimicrobial activity of AgNPs. Therefore, the size and shape of AgNPs were characterized by SEM. Figure 5 shows the SEM images of the synthesized AgNPs. SEM results showed that the synthesized AgNPs size was below $100 \mathrm{~nm}$, which is in a good agreement with DLS results.
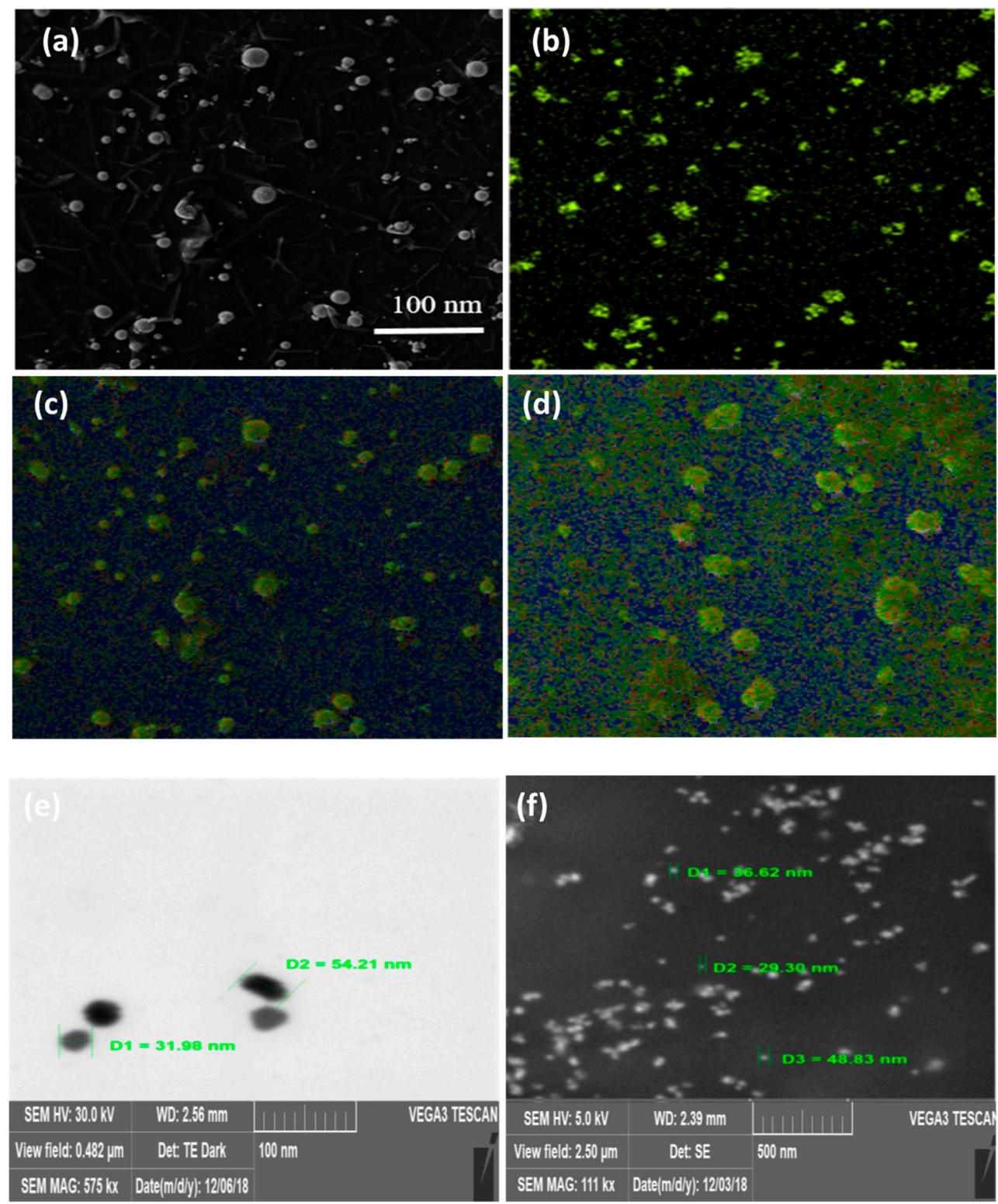

Figure 5. The scanning electron microscopy (SEM) measurements of (a) AgNPs with Cap (b) AgNPs with Gar (c) AgNPs with Gin (d) AgNPs with Cap-Gar (e) AgNPs with Cap-Gin (f) AgNPs with Gar-Gin.

All AgNPs were spherical and polydisperse as indicated by the PDI values. This can be attributed to differences in growth rates of individual particles during the nucleation step. However, some AgNP aggregates and particle size variations were noticed, which may be due to solvent evaporation during sample preparation. A pale, thin layer was seen around the AgNPs, which confirmed the capping 
of the formed nanoparticles by other plant constituents, such as proteins and flavonoid compounds, preventing them from aggregation as evidenced by EDX and FTIR results.

\subsection{Elemental Analysis by EDX}

EDX was also used to reveal the metal composition of AgNPs. The elemental constituents of the AgNPs were also measured by EDX (Figure 6). A strong absorption peak at $3 \mathrm{keV}$ proved the presence of elemental silver in the nanoparticles. The EDX peaks corresponding to carbon, oxygen and sulfur suggest that the AgNPs were successfully capped by plant constituents.

(a)
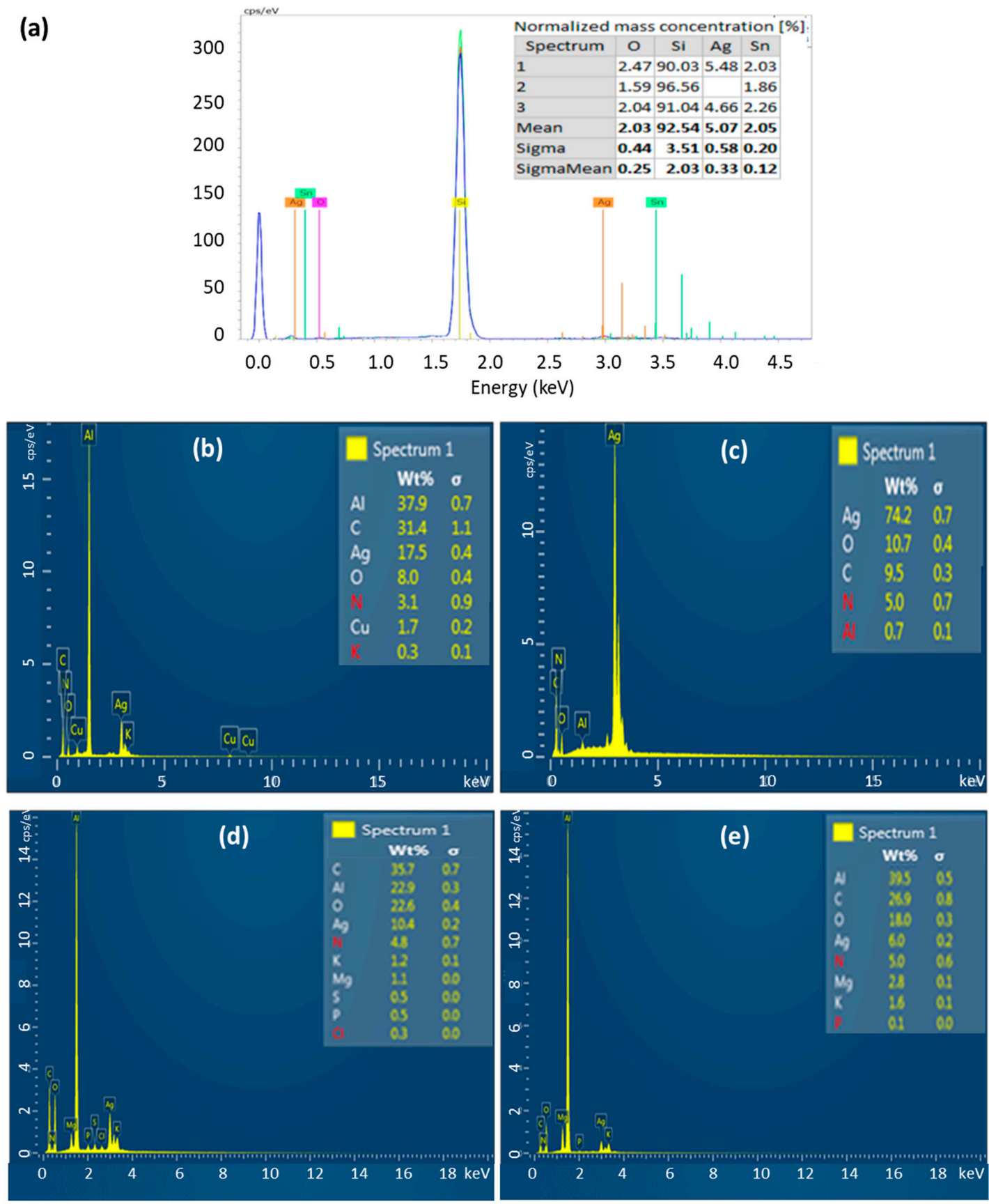

Figure 6. The energy-dispersive X-ray (EDX) measurements of (a) AgNPs with Cap (b) AgNPs with Gar (c) AgNPs with Gin (d) AgNPs with Cap-Gar (e) AgNPs with Cap-Gin. 


\subsection{Fourier-Transform Infrared (FTIR) Spectroscopy}

FTIR spectrum (Figure 7) of the reduced $\mathrm{AgNO}_{3}$ solution with the plants extracts was obtained between the wave number ranges of $4000-600 \mathrm{~cm}^{-1}$. FTIR analyses were performed at a resolution of $4 \mathrm{~cm}^{-1}$ to reveal the potential functional groups in plant extracts that are responsible for reducing the silver ion and capping the formed AgNPs. The appearance of FTIR peak at $3350 \mathrm{~cm}^{-1}$ is indicative of the presence of N-H bond of amines, while the small peak at $2913 \mathrm{~cm}^{-1}$ corresponds to $\mathrm{H}-\mathrm{C}-\mathrm{H}$ symmetric stretching of alkanes. The alkaloid capsaicin with $\mathrm{N}-\mathrm{H}$ stretch is responsible for the bio-reduction of silver ions and acts as a capping agent for the nanoparticles. The band at $1017 \mathrm{~cm}^{-1}$ belongs to C-O stretching of ethers in plant extract. Other phenolic and flavonoid constituents could react with silver ions during the formation of AgNPs [16].

(a)

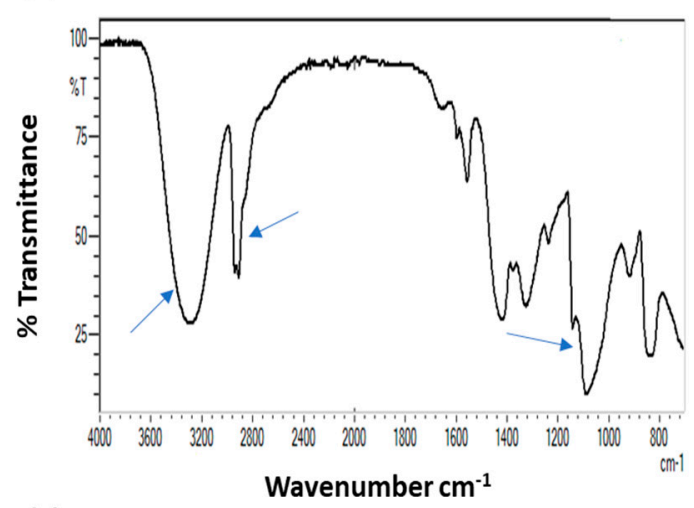

(c)

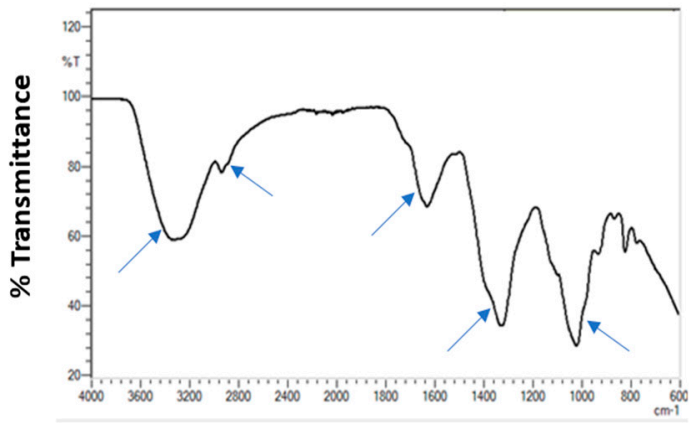

(e)

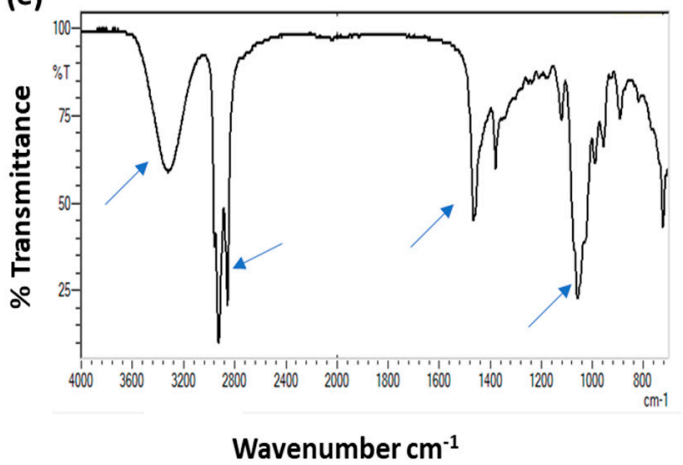

(b)

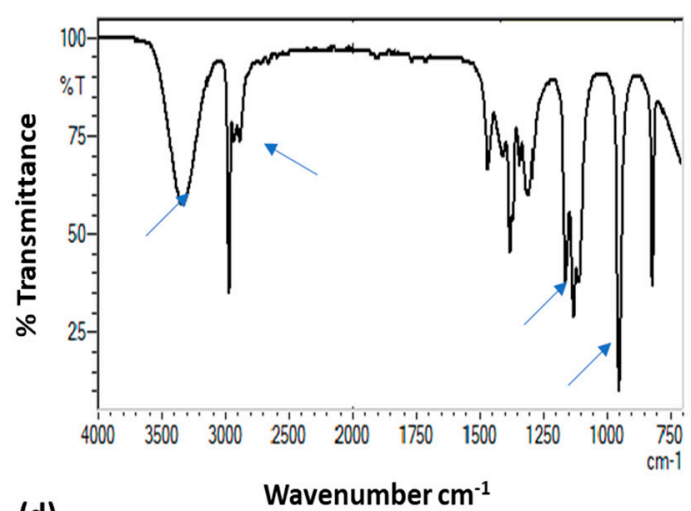

(d)

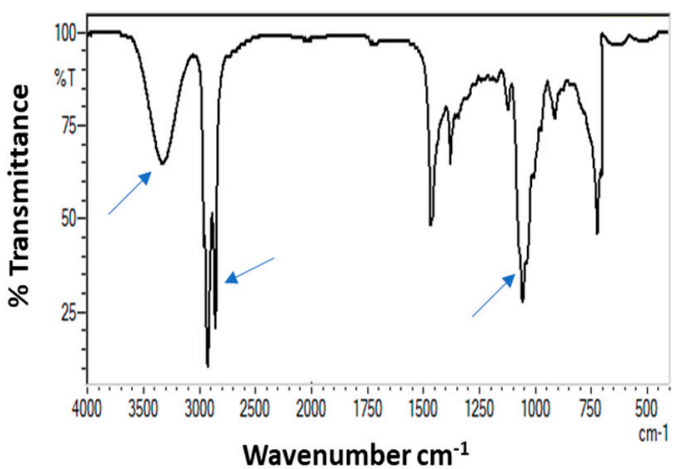

(f)

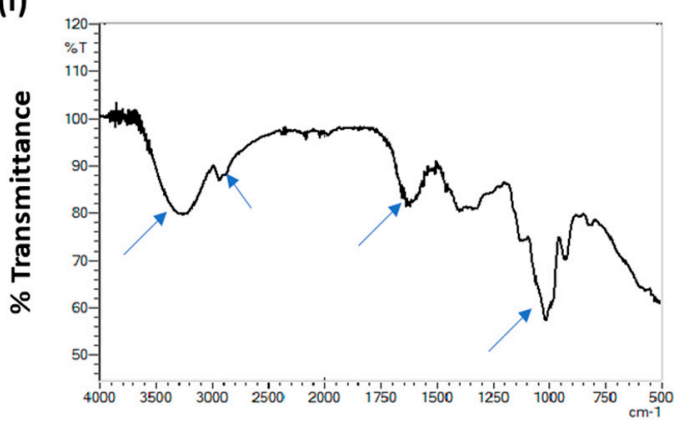

Wavenumber $\mathrm{cm}^{-1}$

Figure 7. Fourier transform infrared (FTIR) of AgNPs: (a) AgNPs with Cap (b) AgNPs with Gar (c) AgNPs with Gin (d) AgNPs with Cap-Gar (e) AgNPs with Cap-Gin (f) AgNPs with Gar-Gin.

In the AgNPs prepared from garlic extract, the observed stretching peaks at 3270 and $2930 \mathrm{~cm}^{-1}$ likely belong to $-\mathrm{O}-\mathrm{H}$ and $-\mathrm{C}-\mathrm{H}$ peaks, respectively, which correspond to sugars in the garlic extract. It is known that garlic contains sugars, such as sucrose and fructose, that act as reducing and stabilizing 
agents [21]. The FTIR of nanoparticles synthesized by using garlic extract was characterized by peaks at 1032,1377 and $2800 \mathrm{~cm}^{-1}$, which correspond to $\mathrm{C}-\mathrm{O}$ (ethers), $\mathrm{C}-\mathrm{H}$ and $\mathrm{C}-\mathrm{S}$, respectively. These groups are present in the active constituents of garlic extract, such as allicin and allyl cysteine. However, the peak at $3450 \mathrm{~cm}^{-1}$ corresponds to the $\mathrm{C}-\mathrm{N}$ of amine.

The FTIR of the ginger extract and AgNPs dispersion showed peaks at 3405, 2800, 1713, 1362 and $1083 \mathrm{~cm}^{-1}$. The strong band at $3405 \mathrm{~cm}^{-1}$ corresponds to strong stretching vibrations of the $\mathrm{O}-\mathrm{H}$ and $\mathrm{C}-\mathrm{N}$ groups. The corresponding peaks at $2800 \mathrm{~cm}^{-1}$ were due to $\mathrm{C}-\mathrm{H}$ stretching vibrations, whereas the peaks at 1713 and $1362 \mathrm{~cm}^{-1}$ were attributed to the $\mathrm{C}=\mathrm{O}$ stretching vibration of ester and alkane group in the active ingredients of ginger [18]. The peak at $1080 \mathrm{~cm}^{-1}$ was attributed to the $\mathrm{O}-\mathrm{H}$ deformation vibration of tertiary $\mathrm{C}-\mathrm{O}-\mathrm{H}$ groups.

The proposed mechanism of the green synthesis of AgNPs can be illustrated as follows:

Active constituent contains- $\mathrm{OH}$ group $+\mathrm{Ag}^{+2} \rightarrow$ active constituent with $=\mathrm{O}$ group $+\mathrm{AgNPs}$

\subsection{In Vitro Antimicrobial Activity of AgNPs}

The antimicrobial activity of AgNPs with different plant capping agents was tested against the pathogens C. albicans, the gram-negative bacteria E. coli and the gram-positive bacteria S. aureus using the agar well diffusion assay. The means of the ZOIs of the three replicate antibacterial assays were recorded (Table 2) for each organism and are shown graphically in Figure 8. AgNPs/plant extract mixtures at a concentration of $50 \mu \mathrm{g} / \mathrm{mL}$ showed high inhibitory effects against the three selected strains. In the present study, the Cap-Gar/AgNPs gave best results against $E$. coli $(\mathrm{ZOI}=30 \pm 0.26 \mathrm{~mm})$. Whereas, the highest activity in the case of $S$. aureus was recorded with Gin-Cap/AgNPs $(Z O I=33 \pm 0.29 \mathrm{~mm}$ ). Regarding the antifungal activity, the Gar-Gin/AgNPs showed the maximum effect against $C$. albicans $(\mathrm{ZOI}=23 \pm 0.33 \mathrm{~mm}$ ) as shown in (Figure 9, Table 2). The results confirmed that the AgNPs synthesized by using the mixture of plant extracts showed more potent antimicrobial activity than the AgNPs synthesized by using pure plant extracts $[19,29,30]$.

Table 2. Antibacterial and antifungal activity of the silver nanoparticles.

\begin{tabular}{ccccc}
\hline \multicolumn{2}{c}{ Silver Nanoparticle Samples } & \multicolumn{2}{c}{ ZOI $(\mathbf{m m})$ against Pathogenic Bacteria } \\
\hline Sample No. & Plant Extract(s) & E. coli & S. aureus & C. albicans \\
\hline 1 & Cap & $22 \pm 0.12$ & $27 \pm 0.41$ & $12 \pm 0.36$ \\
2 & Gar & $22 \pm 0.44$ & $24 \pm 0.16$ & $11 \pm 0.19$ \\
3 & Gin & $21 \pm 0.53$ & $26 \pm 0.32$ & $11 \pm 0.48$ \\
4 & Cap-Gar & $30 \pm 0.26$ & $24 \pm 0.48$ & $25 \pm 0.20$ \\
5 & Gin-Cap & $23 \pm 0.09$ & $33 \pm 0.29$ & $22 \pm 0.53$ \\
6 & Gar-Gin & $20 \pm 0.05$ & $30 \pm 0.36$ & $23 \pm 0.33$ \\
\hline
\end{tabular}

Experiments for plant extracts (without AgNPs) and the $\mathrm{AgNO}_{3}$ solution were performed and no inhibition zone was found in case of plant extract and a mild effect was found in case of $\mathrm{AgNO}_{3}$ solution. The results clearly showed that the inhibitory effect of AgNPs strongly depended on the plant extract used rather than the size of AgNPs. Mixtures of these extracts with AgNPs have even higher antimicrobial activity than pure plant extracts with AgNPs. We can conclude that using more than one plant extract increases the antimicrobial activity of the AgNPs.

Further, the activity of AgNPs against the gram-positive bacteria S. aureus was extraordinary compared to the previous reports in the literature [16,19]. Synergistic antibacterial effects of AgNPs and naturally occurring compounds in capsicum, garlic and ginger possibly resulted in higher bactericidal activity. Numerous naturally occurring ligands, such as proteins, tannin, terpenoids and flavonoids present in the extracts, can interact with the microbial membrane $[13,14]$. 

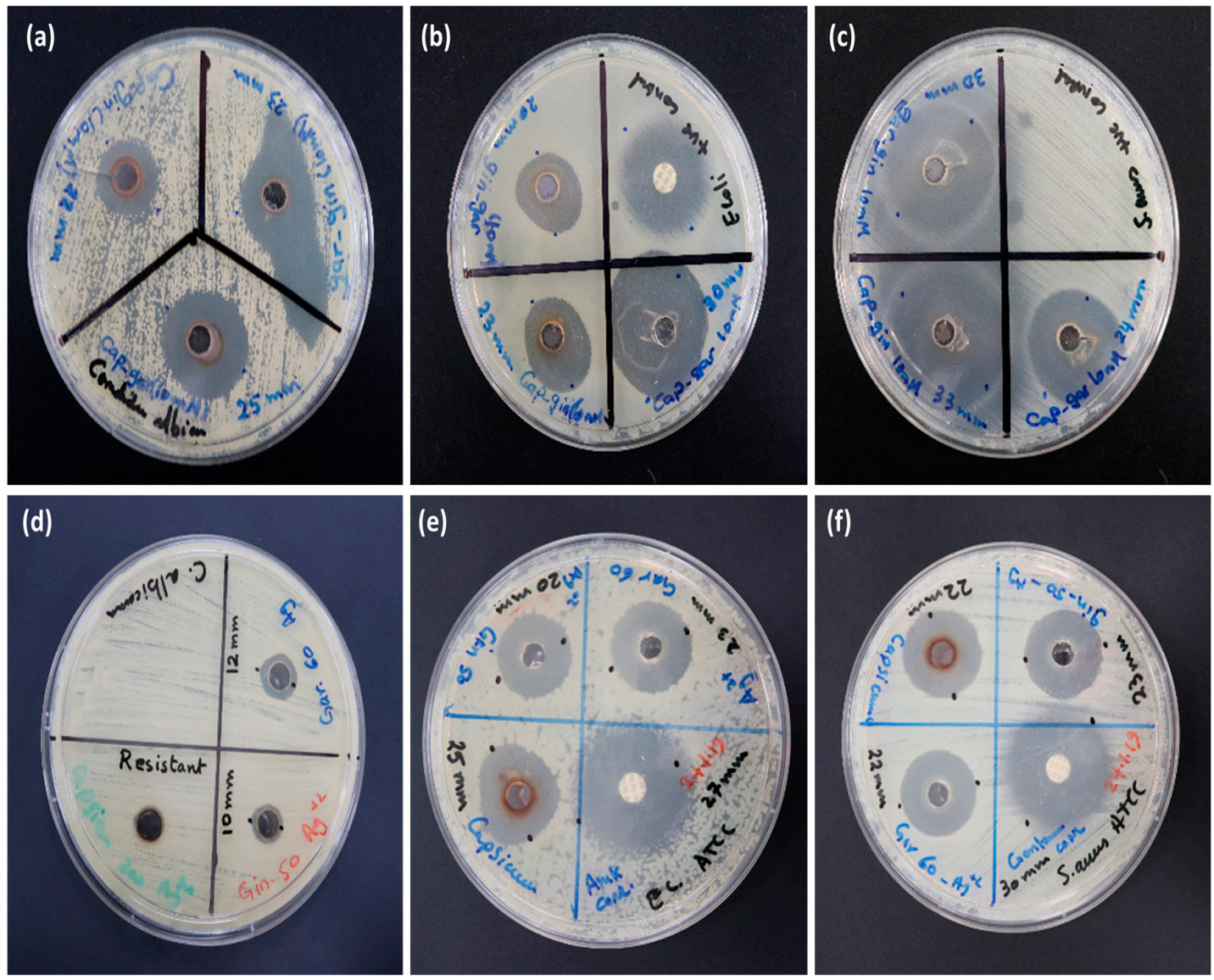

Figure 8. Activity of (a) AgNPs with Cap (b) AgNPs with Gar (c) AgNPs with Gin (d) AgNPs with Cap-Gar (e) AgNPs with Cap-Gin (f) AgNPs with Gar-Gin.

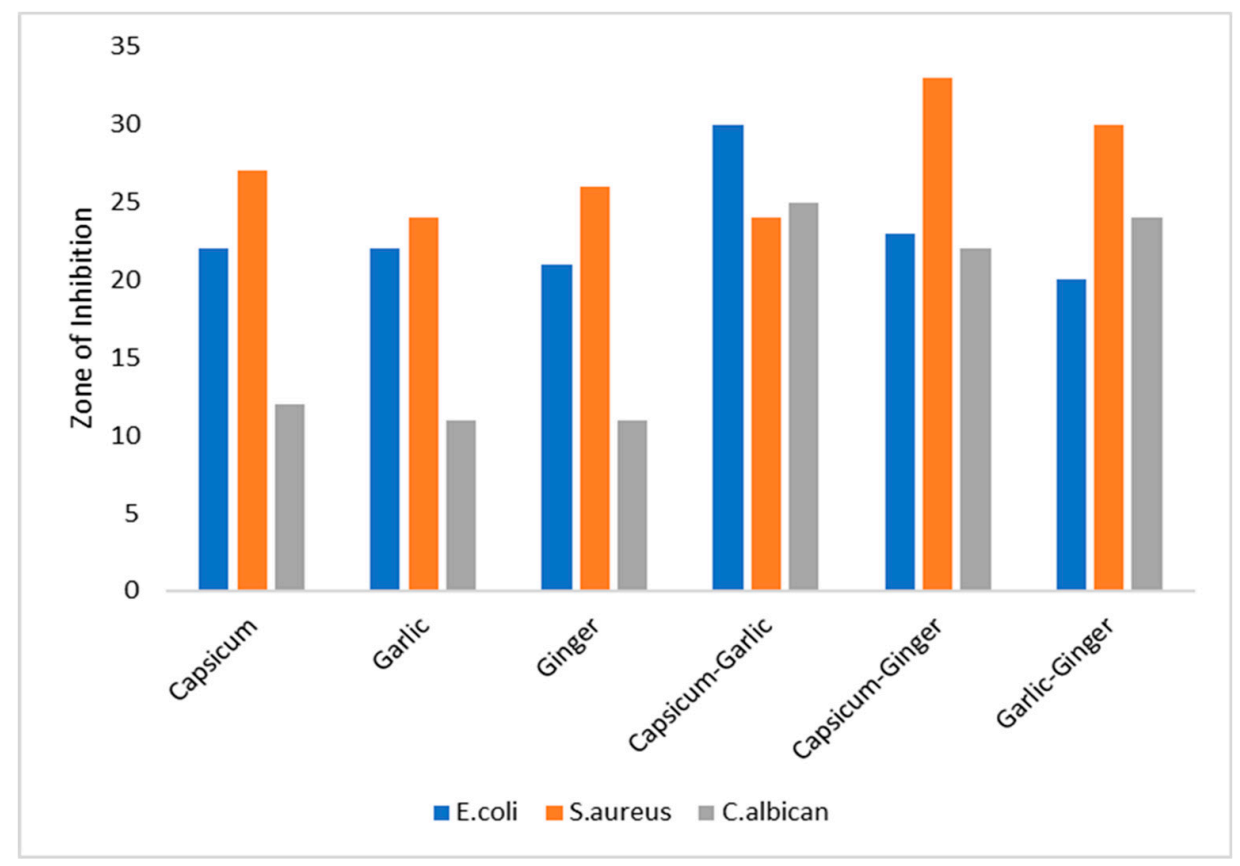

Figure 9. Antimicrobial activity of AgNPs produced from different plant extracts.

Several mechanisms have been reported to explain the lethal effect of AgNPs. This might involve the release of $\mathrm{Ag}^{+}$ions from AgNPs that may attach to the positive charged cell wall, thus leading to 
the deformation of the proteins and resulting in cell death [5]. On the contrary, some studies suggest that the growth inhibition around the well is due to the release of diffusible inhibitory reactive oxygen species (ROS) from AgNPs. The free radicals from metals may damage the bacterial membranes, mitochondria and DNA, which can subsequently cause bursting and death of the cell [21]. Different studies confirmed that green synthesized AgNPs show high antifungal activities against various types of fungi, such as Rhizoctonia solani [25], C. albicans and C. tropicalis [31].

Based on published works, it was concluded that AgNPs could act on cell membranes by destabilizing the cell membrane of bacterial and fungal species. Moreover, AgNPs can also penetrate into cytoplasm and interact with sulfur-containing proteins and phosphorus-containing compounds to interfere with DNA replication [32-34].

Moreover, gram-positive bacteria were significantly $(p<0.001)$ more sensitive to AgNPs compared with gram-negative bacteria. The variation in the effect of AgNPs on gram-negative bacteria and gram-positive bacteria may be due to the difference in the cell wall structure of the two types of bacteria. In case of gram-positive bacteria, there is no outer membrane in the cell wall. However, they both interact diversely with the charged AgNPs $[23,33]$.

\section{Conclusions}

In the present work, we have demonstrated an eco-friendly, simple and low-cost green synthesis method of AgNPs by using various plant extracts as reducing and stabilizing agents. Our investigation provided evidence that synthesized AgNPs/plant extract are very promising candidates that can be used for future biomedical applications. We studied the optical, structural, surface morphology, stability and antibacterial activity of the AgNPs. The DLS studies indicated that the sizes were ranged between 20 and $70 \mathrm{~nm}$, which was confirmed with the SEM measurements. Moreover, FTIR and EDX verified the presence of biological compounds of plant origin surrounding the AgNPs. The synthesized AgNPs using different extracts prepared from different plants including ginger, garlic, capsicum and their mixtures exhibited an excellent antimicrobial activity against $E$. coli and S. aureus and a mild effect on C. albicans.

Author Contributions: A.A. designed the research and M.R. and H.A.S. performed the experiments and analyzed the data. M.R. wrote the manuscript. A.A., Z.E., S.B. and R.B. contributed significantly in revising the final manuscript.

Funding: The work was funded by Ajman University.

Acknowledgments: Authors would like to thank Ajman University for providing financial support to accomplish the present work.

Conflicts of Interest: The authors declare no conflict of interest.

\section{Abbreviations}

$\begin{array}{ll}\text { AgNPs } & \text { Silver nanoparticles } \\ \text { DLS } & \text { Dynamic light scattering } \\ \text { UV-Vis } & \text { Ultra violet visible } \\ \text { FTIR } & \text { Fourier-Transform Infrared Radiometer } \\ \text { SEM } & \text { Scanning light microscope } \\ \text { ZOI } & \text { Zone of inhibition } \\ \text { EDX } & \text { Energy dispersed X-ray } \\ \text { PDI } & \text { Poly-dispersity Index } \\ \text { Gin } & \text { Ginger } \\ \text { Gar } & \text { Garlic } \\ \text { Cap } & \text { Capsicum } \\ \text { Gar-Gin } & \text { Garlic-Ginger } \\ \text { Gin-Cap } & \text { Ginger-capsicum } \\ \text { Cap-Gar } & \text { Capsicum-garlic } \\ \text { NPs } & \text { Nanoparticles } \\ \text { PVA } & \text { Polyvinyl alcohol }\end{array}$




$\begin{array}{ll}\text { PAA } & \text { Polyacrylic acid } \\ \text { MSA } & \text { Mercaptosuccinic acid } \\ \text { PEG } & \text { Polyethylene glycol } \\ \text { ANOVA } & \text { Analysis of variance } \\ \text { SPR } & \text { Surface plasmonic resonance } \\ \text { MHB } & \text { Mueller Hinton Broth } \\ \text { MHA } & \text { Mueller Hinton Agar }\end{array}$

\section{References}

1. Ashour, A.A.; Raafat, D.; El-Gowelli, H.M.; El-Kamel, A.H. Green synthesis of silver nanoparticles using cranberry powder aqueous extract: Characterization and antimicrobial properties. Int. J. Nanomed. 2015, 10, 7207-7221.

2. Chernousova, S.; Epple, M. Silver as antibacterial agent: Ion, nanoparticle, and metal. Angew. Chem. Int. Ed. 2013, 52, 1636-1653. [CrossRef] [PubMed]

3. Li, W.-R.; Xie, X.-B.; Shi, Q.-S.; Zeng, H.-Y.; Ou-Yang, Y.-S.; Chen, Y.-B. Antibacterial activity and mechanism of silver nanoparticles on Escherichia coli. Appl. Microbiol. Biotechnol. 2009, 85, 1115-1122. [CrossRef] [PubMed]

4. Morones-Ramirez, J.R.; Winkler, J.A.; Spina, C.S.; Collins, J.J. Silver Enhances Antibiotic Activity Against Gram-negative Bacteria. Sci. Transl. Med. 2013, 5, 190ra81. [CrossRef] [PubMed]

5. Kim, K.-J.; Sung, W.S.; Suh, B.K.; Moon, S.-K.; Choi, J.-S.; Kim, J.G.; Lee, D.G. Antifungal activity and mode of action of silver nano-particles on Candida albicans. BioMetals 2008, 22, 235-242. [CrossRef]

6. Pozdnyakov, A.S.; Emel'Yanov, A.I.; Kuznetsova, N.P.; Ermakova, T.G.; Fadeeva, T.V.; Sosedova, L.M.; Prozorova, G.F. Nontoxic hydrophilic polymeric nanocomposites containing silver nanoparticles with strong antimicrobial activity. Int. J. Nanomed. 2016, 11, 1295-1304. [CrossRef]

7. Jaiswal, S.; Bhattacharya, K.; McHale, P.; Duffy, B. Dual effects of $\beta$-cyclodextrin-stabilised silver nanoparticles: Enhanced biofilm inhibition and reduced cytotoxicity. J. Mater. Sci. Mater. Electron. 2015, 26, 52. [CrossRef]

8. Ajitha, B.; Reddy, Y.A.K.; Reddy, P.S.; Jeon, H.-J.; Ahn, C.W. Role of capping agents in controlling silver nanoparticles size, antibacterial activity and potential application as optical hydrogen peroxide sensor. RSC Adv. 2016, 6, 36171-36179. [CrossRef]

9. Wei, L.; Lu, J.; Xu, H.; Patel, A.; Chen, Z.S.; Chen, G. Silver nanoparticles: Synthesis, properties, and therapeutic applications. Drug Discov. Today 2015, 20, 595-601. [CrossRef]

10. Rafique, M.; Sadaf, I.; Rafique, M.S.; Tahir, M.B. A review on green synthesis of silver nanoparticles and their applications. Artif. Cells Nanomed. Biotechnol. 2017, 45, 1272-1291. [CrossRef]

11. Sathishkumar, M.; Sneha, K.; Won, S.; Cho, C.-W.; Kim, S.; Yun, Y.-S. Cinnamon zeylanicum bark extract and powder mediated green synthesis of nano-crystalline silver particles and its bactericidal activity. Colloids Surf. B Biointerfaces 2009, 73, 332-338. [CrossRef] [PubMed]

12. Khatami, M.; Pourseyedi, S. Phoenix dactylifera (date palm) pit aqueous extract mediated novel route for synthesis high stable silver nanoparticles with high antifungal and antibacterial activity. IET Nanobiotechnol. 2015, 9, 184-190. [CrossRef] [PubMed]

13. Mohan Kumar, K.; Siva Kumar, K.; Sinha, M.; Mandal, B.K.; Sreedhara Reddy, P.; Ghosh, A.R. Green synthesis of silver nanoparticles using Terminalia chebula extract at room temperature and their antimicrobial studies. Spectrochim. Acta Part A Mol. Biomol. Spectrosc. 2012, 91, 228-233. [CrossRef] [PubMed]

14. Reddy, N.J.; Vali, D.N.; Rani, M.; Rani, S.S. Evaluation of antioxidant, antibacterial and cytotoxic effects of green synthesized silver nanoparticles by Piper longum fruit. Mater. Sci. Eng. C 2014, 34, 115-122. [CrossRef] [PubMed]

15. Sharma, V.K.; Yngard, R.A.; Lin, Y. Silver nanoparticles: Green synthesis and their antimicrobial activities. Adv. Colloid Interface Sci. 2009, 145, 83-96. [CrossRef] [PubMed]

16. Shankar, T.; Karthiga, P.; Swarnalatha, K.; Rajkumar, K. Green synthesis of silver nanoparticles using Capsicum frutescens and its intensified activity against E. coli. Resour. Technol. 2017, 3, 303-308.

17. Otunola, G.A.; Afolayan, A.J.; Ajayi, E.O.; Odeyemi, S.W. Characterization, antibacterial and antioxidant properties of silver nanoparticles synthesized from aqueous extracts of Allium sativum, Zingiberofficinale, and Capsicum frutescens. Pharmacogn. Mag. 2017, 13, 201-208. [CrossRef] 
18. Yang, N.; Li, F.; Jian, T.; Liu, C.; Sun, H.; Wang, L.; Xu, H. Biogenic synthesis of silver nanoparticles using ginger (Zingiberofficinale) extract and their antibacterial properties against aquatic pathogens. Acta Oceanol. Sin. 2017, 36, 95-100. [CrossRef]

19. El-Refai, A.A.; Ghoniem, G.A.; El-Khateeb, A.Y.; Hassaan, M.M. Eco-friendly synthesis of metal nanoparticles using ginger and garlic extracts as biocompatible novel antioxidant and antimicrobial agents. J. Nanostruct. Chem. 2018, 8, 71-81. [CrossRef]

20. Chung, L.Y. The Antioxidant Properties of Garlic Compounds: Allyl Cysteine, Alliin, Allicin, and Allyl Disulfide. J. Med. Food 2006, 9, 205-213. [CrossRef]

21. Von White, G.; Kerscher, P.; Brown, R.M.; Morella, J.D.; McAllister, W.; Dean, D.; Kitchens, C.L. Green synthesis of robust, biocompatible silver nanoparticles using garlic extract. J. Nanomater. 2012, 2012, 55. [CrossRef]

22. Kharissova, O.V.; Dias, H.V.R.; Kharisov, B.I.; Pérez, B.O.; Pérez, V.M.J. The greener synthesis of nanoparticles. Trends Biotechnol. 2013, 31, 240-248. [CrossRef]

23. Govindappa, M.; Hemashekhar, B.; Arthikala, M.K.; Ravishankar Rai, V.; Ramachandra, Y.L. Characterization, antibacterial, antioxidant, antidiabetic, anti-inflammatory and antityrosinase activity of green synthesized silver nanoparticles using Calophyllumtomentosum leaves extract. Results Phys. 2018, 9, 400-408. [CrossRef]

24. Pasupuleti, V.R.; Prasad, T.N.V.K.V.; Shiekh, R.A.; Balam, S.K.; Narasimhulu, G.; Reddy, C.S.; Gan, S.H. Biogenic silver nanoparticles using Rhinacanthusnasutus leaf extract: Synthesis, spectral analysis, and antimicrobial studies. Int. J. Nanomed. 2013, 8, 3355-3364. [CrossRef]

25. Khatami, M.; Mehnipor, R.; Poor, M.H.S.; Jouzani, G.S. Facile Biosynthesis of Silver Nanoparticles Using Descurainiasophia and Evaluation of Their Antibacterial and Antifungal Properties. J. Clust. Sci. 2016, 27, 1601-1612. [CrossRef]

26. Sun, Q.; Cai, X.; Li, J.; Zheng, M.; Chen, Z.; Yu, C.-P. Green synthesis of silver nanoparticles using tea leaf extract and evaluation of their stability and antibacterial activity. Colloids Surf. A Physicochem. Eng. Asp. 2014, 444, 226-231. [CrossRef]

27. Bindhu, M.; Umadevi, M. Synthesis of monodispersed silver nanoparticles using Hibiscus cannabinus leaf extract and its antimicrobial activity. Spectrochim. Acta Part A Mol. Biomol. Spectrosc. 2013, 101, 184-190. [CrossRef]

28. Wang, L.; Wu, Y.; Xie, J.; Wu, S.; Wu, Z. Characterization, antioxidant and antimicrobial activities of green synthesized silver nanoparticles from Psidium guajava L. leaf aqueous extracts. Mater. Sci. Eng. C 2018, 86, 1-8. [CrossRef]

29. Anandalakshmi, K.; Venugobal, J.; Ramasamy, V. Characterization of silver nanoparticles by green synthesis method using Pedalium murex leaf extract and their antibacterial activity. Appl. Nanosci. 2016, 6, 399-408. [CrossRef]

30. Sre, P.R.; Reka, M.; Poovazhagi, R.; Kumar, M.A.; Murugesan, K. Antibacterial and cytotoxic effect of biologically synthesized silver nanoparticles using aqueous root extract of Erythrina indica lam. Spectrochim. Acta Part A Mol. Biomol. Spectrosc. 2015, 135, 1137-1144.

31. Mallmann, E.J.J.; Cunha, F.A.; Castro, B.N.; Maciel, A.M.; Menezes, E.A.; Fechine, P.B.A. Antifungal Activity of Silver Nanoparticles Obtained by Green Synthesis. Rev. Inst. Med. Trop. Sao Paulo 2015, 57, 165-167. [CrossRef]

32. Mariselvam, R.; Ranjitsingh, A.J.A.; Usha Raja Nanthini, A.; Kalirajan, K.; Padmalatha, C.; MosaeSelvakumar, P. Green synthesis of silver nanoparticles from the extract of the inflorescence of Cocos nucifera (Family: Arecaceae) for enhanced antibacterial activity. Spectrochim. Acta Part A Mol. Biomol. Spectrosc. 2014, 129, 537-541. [CrossRef]

33. Bindhu, M.; Umadevi, M. Antibacterial and catalytic activities of green synthesized silver nanoparticles. Spectrochim. Acta Part A Mol. Biomol. Spectrosc. 2015, 135, 373-378. [CrossRef]

34. Tippayawat, P.; Phromviyo, N.; Boueroy, P.; Chompoosor, A. Green synthesis of silver nanoparticles in aloe vera plant extract prepared by a hydrothermal method and their synergistic antibacterial activity. PeerJ 2016, 4, e2589. [CrossRef]

(C) 2019 by the authors. Licensee MDPI, Basel, Switzerland. This article is an open access article distributed under the terms and conditions of the Creative Commons Attribution (CC BY) license (http://creativecommons.org/licenses/by/4.0/). 